\title{
A Cultural Challenge: Teaching Mathematics to Non-mathematicians
}

ANDRIJANA D. BURAZIN, Department of Mathematical and Computational Sciences, University of Toronto Mississauga, Canada

VESELIN JUNGIC, Department of Mathematics, Simon Fraser University, Canada

MIROSLAV LOVRIC, Department of Mathematics and Statistics, McMaster University, Canada

\begin{abstract}
A "mathematics for non-mathematicians" course, commonly known as a "service" course is an undergraduate mathematics course developed for students who are not (going to become) mathematics majors. Besides calculus, such courses may include linear algebra, mathematical reasoning, differential equations, mathematical programming and modeling, discrete mathematics, mathematics for teachers, and so on. In this article we argue that a good, productive curricular design and teaching of service courses happen through a meaningful collaboration between a mathematics instructor and the department whose students are taking the course. This collaboration ensures that "non-mathematicians" see the relevance of learning mathematics for their discipline (say, by discussing authentic problems and examples), but also appreciate the relevance and benefits which mathematics brings to their overall education and skills set.
\end{abstract}

Additional Keywords and Phrases: non-mathematicians, service courses, collaborative curriculum, authentic applications

\section{Recommended Reference Format:}

Andriana D. Burazin, Veselin Jungic, and Miroslav Lovric. 2021. A Cultural Challenge: Teaching Mathematics to Nonmathematicians. Maple Trans. 1, 1, Article 14144 (July 2021), 11 pages.

https://doi.org/10.5206/mt.v1i1.14144

\section{Introduction}

Does a university mathematics instructor need to be a mathematician?

What a silly question! Isn't the answer, obviously, yes?

We, three mathematicians with extensive experience teaching mathematics, feel that we need to consider some possibly counterintuitive factors before trying to answer this question.

From our own experience, we know that mathematicians are heavily biased towards their beloved subject. This bias is part of common "attitudes, behaviours and values" among members of the academic mathematical community that Leone Burton calls "mathematical culture" [4]. In the same article she states:

"It is a major irony that, while the mathematicians learn in an enquiry style, they revert to traditional teaching practices with their students. But this means they carry with them into their classrooms some of the worst features of the mathematical culture, in particular the reification of hierarchies in learning, competitive practices and isolation." [4]

Burton's statement reinforces our concerns that mathematicians should not be trusted to teach mathematics, in disciplinary isolation, to those students who do not plan to become professional mathematicians.

This is one of the reasons why, with this article, we are joining a growing community of academics who are arguing for an interdisciplinary approach to teaching mathematics for nonmathematicians, and for a mutually beneficial integration of mathematics and the discipline for which the mathematics content is taught. For example, in 2015, AMATYC (American 
Mathematical Association of Two-Year Colleges), AMS (American Mathematical Society), ASA (American Statistical Association), MAA (Mathematical Association of America), and SIAM (Society for Industrial and Applied Mathematics) jointly sponsored "The Common Vision" project with the ultimate goal to

"galvanize the mathematical sciences community around a modern vision for undergraduate programs and to spur grassroots efforts within the community as a foundation for addressing the collective challenges we face." [14]

Among the conclusions of this project we find the following recommendation:

"Mathematicians should seek (1) applications from partner disciplines for use in mathematics courses, (2) team teaching opportunities with faculty in partner disciplines, and (3) input from business and industry on desired workplace skills. They should collaborate with partner disciplines to explicitly translate notation and terminology among the disciplines in order to enhance students' ability to see connections across disciplines." [14]

Simply stated, designing and teaching a mathematics course for non-mathematicians calls for collaboration between mathematics and instructors from the discipline, be it physics, economics, or fine arts.

Why do we need to change the way we teach mathematics for non-mathematicians? For the authors of this article, it is increasingly clear that such change is necessary to ensure that mathematics survives outside of mathematics departments. In our view, the discipline of abstract mathematics is, and has always been, in good shape, with strong research, exciting new discoveries, and emergent new areas. But we are not so sure what the future will bring to mathematics for non-mathematicians, and who will, or should, have control over this branch of mathematics in terms of curriculum and teaching.

In [12], the author states that teaching mathematics to non-mathematicians is an emerging area of research in mathematics education, which is trying to address a multitude of related issues, including

"double discontinuity between school, university and workplace mathematics and the challenges of teaching mathematical modelling to students from within and from outside mathematics."

In what follows, we describe so-called "service mathematics" courses and reflect about their role in the post-secondary education. We also offer several examples and ideas on how to make such courses more interdisciplinary, and thus more relevant and viable in the long-term.

Furthermore, we argue that, for the benefit of all stakeholders, mathematics courses for nonmathematicians should play the role of a bridge not only between expertise and bodies of knowledge but also between academic cultures.

\section{Service Courses}

A service course is a collective term for an undergraduate mathematics course developed for students in non-mathematics programs. Besides calculus, such courses may include linear 
algebra, mathematical reasoning, differential equations, mathematical programming and modeling, discrete mathematics, mathematics for teachers, and so on.

Commonly, students in these courses are not exposed to the level of rigor and formalism that characterize academic mathematics. For example, they are rarely shown rigorous mathematical proofs and are seldom, if ever, asked to demonstrate their ability to reproduce such a proof.

Mathematics service courses are expected to achieve multiple goals: from acting as gate keepers for certain programs, to functioning as a "check box" in the specific program accreditation process, to serving as genuine and essential program requirements.

Because of the large numbers of students taking these courses, service courses are important income generator for departments and faculties. For example, in 2019-20, the Department of Mathematics at Simon Fraser University (SFU) in Burnaby, British Columbia, Canada, reported the total of activity full time equivalent (AFTE) enrolment of 1,077.4. At the same time, there were 425 students enrolled in one of the Department's major or minor programs. For the same school year, the largest department in the SFU's Faculty of Science, the Department of Biomedical Physiology \& Kinesiology, reported a total AFTE enrolment of 855 with the total of 1,086 students enrolled in one of the Department's major or minor programs. This somewhat stunning disproportion comes from the number of students taking one of the 16 service courses offered by the Department of Mathematics.

\section{Teaching Service Courses}

"I have often been struck with the dry, uninteresting manner in which various subjects are being treated. The examples are mostly abstract, very few practical problems to work out. Unless the student is very intelligent, his mind retains nothing beyond a chaos of formulae hard to remember and a few mechanical means of solving abstract problems. He is incapable of applying an equation to a practical problem." [11]

This quote was taken from the paper entitled "The Teaching of Mathematics to Students of Engineering: From the Standpoint of the Practising Engineer," which criticized the trends in the teaching of mathematics to future engineers.

A brief glance at any randomly chosen mathematics textbook provides evidence that (except for the idiosyncrasies of the time) very little has substantially changed since this article appeared in Science. "The examples are mostly abstract, very few practical problems to work out" remains true today, as much as it did 110 years ago. The ways we teach mathematics have, at least on the surface, evolved. Calculus has become the "go-to" mathematics course for most first-year students, irrespective of their program of study. Universities offer calculus courses with different flavours: Calculus for Engineering, Calculus for Life Sciences, Calculus for Business and Economics, and so on.

In our experience, mathematics instructors who teach service courses rarely, if ever, communicate with the departments whose students enrol in these courses. Often, they do not make an effort to demonstrate, through authentic, real-world examples and problems, how the mathematics content they teach can be applied. The reasons for this situation range from beliefs that mathematics is a study of abstract systems, where so-called real-life applications have no 
place, to instructors not being knowledgeable enough about the service discipline, to the inability to confidently present discipline-related problems.

The gap between what students see in their mathematics courses and in their program-specific courses is, in our view, the main reason why many of them feel disconnected from mathematics in general. They take mathematics because it is a requirement for their degree, suffer through the "chaos of formulae hard to remember and a few mechanical means of solving abstract problems," as the authors of [11] put it, and leave, at best, with a few vague ideas about mathematics and very little practical experience. To be fair to our students, we observe that their attitude and negative experiences may come from earlier exposures to mathematics. A mathematician and popular science writer Keith Devlin described his generation's experience (mid-1960s) with pre-university mathematics as frustrating, to say the least:

The majority of my fellow K-12 schoolmates not only never got to the stage of making that transition [from the "drill the basics" approach] to mathematical thinking, they ended up hating math (in some cases being very afraid of it), and dropping it at the first opportunity. Even worse, they ended up with a perception of what mathematics is that is dangerously wrong (...). [5]

To acknowledge the immense challenges faced by instructors assigned to teach fast-paced and topic-packed service courses to large and diverse audiences of students, we mention our own frustration with the high level of resistance encountered when we try to help our students transition to mathematical thinking. A recent experience, possibly due to the effects of the pandemic at the time of writing, lead us to think that some students took it as a personal offense when we asked them to step outside of, to paraphrase Devlin, the "drilled basics."

The responsibility to ensure that future generations of scientists, engineers, businesspeople, physicians, teachers, and other professionals, as well as ordinary citizens, are well equipped to learn, understand, and confidently apply mathematical concepts is too large to be put solely on the shoulders of the members of the mathematical guild.

As a piece of evidence that at least some segments of the mathematical community have been listening what others have to say about teaching of mathematics, we acknowledge the MAA project entitled "The Curriculum Foundations Project: Voices of the Partner Disciplines." [10]

We have already witnessed departments across university campuses creating and teaching their own mathematics courses. In most cases, our colleagues from other departments claim that they do not find the courses taught within mathematics departments appropriate or useful for their students.

An example from Wright State University (WSU), Dayton, Ohio, USA, is worth mentioning. The course, EGR 101: Introductory Mathematics for Engineering Applications, a National Science Foundation (NSF) supported initiative, is described as follows:

The WSU model for engineering mathematics education involves the introduction of a novel freshman engineering mathematics course EGR 101, along with a substantial restructuring of the early engineering curriculum. Taught by engineering faculty, the EGR 101 course includes lecture, laboratory and recitation components. Using an applicationoriented, hands-on approach, EGR 101 addresses only the salient math topics actually used in the core entry-level engineering courses. These include the traditional physics, 
engineering mechanics, electric circuits and computer programming sequences. More importantly, the EGR 101 course replaces traditional math prerequisite requirements for the above core courses, so that students can advance in the engineering curriculum without first completing the required calculus sequence. The result has shifted the traditional emphasis on math prerequisite requirements to an emphasis on engineering motivation for math, with a just-in-time structuring of the required math sequence. [8]

The outcomes of offering EGR 101 at WSU for more than a decade are presented in [9]. For example, the authors state that "students who took EGR 101 earned WSU degrees at significantly higher rates than those who did not."

Related to the WSU example, we voice our concerns that the narrowing of the scope of a mathematics course (as a service department may wish to do) could improve the current moment in the life of an academic unit, but in the long run, may threaten the level of mathematical proficiency among students in their programs. We use the notion of computational thinking to illustrate the fact that the ability to learn and apply complex mathematical ideas and techniques are necessary conditions for the further development of sciences.

The term computational thinking was popularized by Wing [16], whose aim was to describe the educational ecosystem that includes computer programming as one of its species. Wing argues that computational thinking complements and combines mathematical and engineering and science thinking, and suggests that, "To reading, writing, and arithmetic, we should add computational thinking to every child's analytical ability."

Here is a widely accepted definition of computational thinking by Aho [1]:

"We consider computational thinking to be the thought processes involved in formulating problems so their solutions can be represented as computational steps and algorithms."

To firmly situate computational thinking and to elaborate on his definition, Aho adds:

"However, as the computer systems we wish to build become more complex and as we apply computer science abstractions to new problem domains, we discover that we do not always have the appropriate models to devise solutions. In these cases, computational thinking becomes a research activity that includes inventing appropriate new models of computation." [1]

How is computational thinking as a "research activity that includes inventing appropriate new models of computation" carried? Aho suggests:

"Mathematical abstractions called models of computation are at the heart of computation and computational thinking." [1]

One of the founders of experimental mathematics, Jonathan Borwein, warns us that "we need to teach procedural or algorithmic thinking" [3]. He leaves no doubt that, in his experience, this is a responsibility of a mathematics teacher.

Combining Aho and Borwein's reflections creates a vision in which mathematics and computing science are strongly intertwined and inseparable. 
In conclusion, neither mathematicians nor non-mathematicians should teach mathematics service courses in isolation from the other. The only way to improve this situation, in our view, lies in the collaboration between departments and curricular co-design of such courses.

\section{The Challenge}

How can we design and teach a mathematics course in which future professionals will get the best of both worlds; in other words, where they will appreciate the width and depth of mathematical concepts and, at the same time, see the reasons why these concepts were developed in the first place through meaningful, authentic applications in the context of their discipline?

The challenge, in our view, is to replace the currently common practice of mathematical training with more meaningful mathematical education. To successfully address this challenge requires a shift in the culture of our academic departments and units.

But what is the culture of a group of people? After asserting that "there is little consensus about the meaning of culture within and across disciplines" [6], Gutiérrez continues:

Hence, while culture can be broadly understood as the patterns of beliefs, values, and practices that we both inherit and transform over time, individuals never share all of the culture of the group to which they belong. At the same time, cultural practices are open or responsive to their ever-changing environment. From this view, culture is both patterned and dramatically varied. [6]

From our experience, we can confirm Gutiérrez's point that culture is "both patterned and dramatically varied" by observing the culture of any scientific field, or any discipline, as "the patterns of beliefs, values, and practices" are in a permanent state of flux.

Consider, for example, the cultural revolution that modern technology has brought to mathematics: a modern mathematician will likely believe in a computationally established mathematical fact even if the formal proof evades them at the moment. Borwein states that:

"[M]odern computational tools dramatically change the nature and scale of available evidence. Given an interesting identity buried in a long and complicated paper on an unfamiliar subject, which would give you more confidence in its correctness: staring at the proof, or confirming computationally that it is correct to 10,000 decimal places?" [3]

Borwein's observation about the shift from primarily relying on proofs to accepting the validity of a computational argument or result reflects the culture change among mathematicians. For several accessible examples that suggest that this kind of the culture change may go back and forth see [7].

In our view, the starting point for the aforementioned shift in the culture of academic departments and units is to recognize that each scientific field and each academic unit have their own culture. In this sense, teaching, as well as taking, a mathematics service course are cross-cultural experiences and, as such, necessary generate culture-conflict situations, as observed by Valero, Christensen, and Skovsmose:

"In university settings, different academic cultures overlap, integrate and also conflict with each other." [15] 
As an outcome, for example, we witness the previously mentioned gap between what students experience in their mathematics courses and in their program-specific courses, such as using different terms for the same object or concept and applying different levels of rigour when establishing evidence.

Culture-conflict situations have a potential to generate positive forces that can contribute to integrating mathematics and service disciplines. We are fully aware that this is a gigantic task whose implementation will face resistance:

"Intertwined with institutional tensions were cultural tensions where the [new] pedagogy was in conflict with the classroom culture that most students were accustomed to. While some students appreciated this change, many students were made uncomfortable by [new] practices." [13]

Still, as claimed in [2], there is a need for the "culture-conflict situation [to be] recognized and curricula re-examined."

\section{Moving Forward}

In this section we offer several ideas and encouraging examples demonstrating that a cultural shift in designing service mathematics courses is possible, and is already happening at some institutions.

As we have already stated, designing and teaching a service mathematics course calls for collaboration between mathematics and service-discipline instructors. This collaboration should start with a conversation about course objectives and include a close examination of the mathematics material that is needed for a specific discipline that uses mathematics. The authors of this article can attest to revealing experiences of looking at the textbooks used in engineering, commerce, economics, life sciences and physics, and extracting mathematics from it. It is quite disturbing to realize how rarely such a simple exercise takes place.

In the Integrated Science Program at McMaster University in Hamilton, Ontario, Canada, instructors in one science discipline visit lectures from other disciplines. For a mathematician, just spending one hour in a physics lecture suffices to reveal the cultural divide between mathematics and physics. Yes, it is mathematics: differentiation, integration, and linear algebra are all there; but physics looks, and feels, for a lack of a better word, different. It is not just the notation; the vocabulary changes, and, most importantly, the approaches to explaining concepts and ideas are not those a mathematician would use. For instance, mathematical formulas used in a physics course are not derived in an abstract vacuum, but within a specific context, which means that they are guided and supported by intuition and knowledge of the physical phenomena involved.

Large parts of mathematics were discovered through attempts to solve real-world problems, or explaining something; for instance, optical field theory and Maxwell equations introduced the vector operations of curl and divergence. Presently, vector operations are taught in a mathematics course usually called "Vector Calculus," where (going back to [11]) "examples are mostly abstract, [and] very few practical problems [are given] to work out."

A natural question arises: why don't we introduce vector operations in a course on electricity and magnetism (mathematics majors would certainly benefit from taking such a course!), rather than expect that they would be covered in a service mathematics course? Add to this observation 
the capacity of Maple, Mathematica, or any other mathematical software used to simulate and visualize almost all mathematical operations, and suddenly a student is in the position to witness that the velocity of a fluid has something to do with how each particle in the fluid behaves.

Interdisciplinary curricular co-design strengthens all courses involved. In mathematics, we rarely mention the word "phase" when talking about the polar form of a complex number. On the other hand, the quantities that have magnitude and phase (sinusoidal voltage, current) are routinely described in engineering and physics using complex numbers. In a service mathematics course, this reality is (if at all) an afterthought. Imagine an enriched view that a mathematics student would have from understanding that the complex numbers are not there solely to compute roots of negative numbers.

A life scientist's evidence is often based on experiments. Then, what is the point of presenting mathematical evidence (a rigid proof) to future biologists, or environmental scientists? A biologist knows that a certain ordinary differential equation that models the growth of a bacteria culture must have a solution, because they have observed it in their experiments!

Why not embrace experiments as evidence in mathematics and use computers and simulations to do it? Instead of teaching abstract theorems, we should teach life sciences students how to code, and then ask them to perform mathematical experiments, verify mathematical claims, and problem-solve. A life scientist is never satisfied with three or four steps in Euler's method (what is typically done in a mathematics lecture), but needs to observe, for instance, what happens in the long term; although formally an infinite limit, "long term" can be achieved by running an algorithm for a sufficient number of steps.

What about mathematics for students in the humanities, economics, or social sciences? A version of a calculus course is often the simplest option, but is it worth it? As an example, a creative alternative is presented by the "Numbers of Life" course taught at McMaster University. In the course, which requires high school mathematics (and no university-level mathematics), students apply their mathematical reasoning to engage with relevant and authentic content drawn from their disciplines. For instance, they learn about crypto currencies, hash functions, and blockchain technology; they understand GDP and its benefits and drawbacks; they calculate and interpret the Gini index of inequality and apply a regression calculator to check the estimates on the disappearance of Arctic ice presented in newspapers. They study contingency tables and principles of medical testing and learn about the communication of probability and risk.

\section{Conclusion}

Does a university mathematics instructor need to be a mathematician?

In this article we argue that designing and teaching a service mathematics course calls for collaboration between mathematics and service-discipline instructors.

Still, for the benefit of students' intellectual and professional growth, mathematical content should remain central, in some form, in all mathematics service courses. This content should be a balanced mixture of a reasonable number of relevant, big ideas and important mathematical procedures. A future engineer, scientist, economist, and teacher should get a fair chance to grasp, for example, the concept of a function, the idea of an infinitesimal, and the notion of a vector. At the same time, in our experience, it is unreasonable to expect that students will be 
able to perform any algorithm without an appropriate step-by-step introduction of mathematical procedures.

What mathematical education provides over mathematical training in the learning process is students' understanding that the learning of mathematics augments their academic growth. This can be achieved through the meaningful motivation of mathematical ideas and procedures, their presentation at an appropriate level of rigour, and their authentic applications.

By overlapping two academic cultures, one of mathematics and the other of a student's field of study, the course instructor can make the value of mathematical knowledge "explicit and overt" [2]. The time has come for mathematicians as instructors of service courses to open their minds, view their subject through an interdisciplinary lens, and to modify what they teach - not just for their students' benefit, but for the benefit of mathematics as well.

\section{Acknowledgments}

We would like to thank Dr. Ozren Jungic for his contribution to improving the text.

\section{References}

[1] Alfred V. Aho. (2012). Computation and computational thinking. Computer Journal 55, 832835.

[2] Alan J. Bishop. (1988). Mathematics Education in Its Cultural Context. Educational Studies in Mathematics 19(2), 179-191.

[3] Jonathan M. Borwein. (2008). Implications of experimental mathematics for the philosophy of mathematics. In Proof and Other Dilemmas: Mathematics and Philosophy. Providence, RI: Amer. Math. Soc., 33-61.

[4] Leone Burton. (2009). The Culture of Mathematics and the Mathematical Culture. In University Science and Mathematics Education in Transition. New York, NY: Springer.

[5] Keith J. Devlin. (2019). Mathematics Is a Way of Thinking - How Can We Best Teach It? Mathematical Association of America. https://www.mathvalues.org/masterblog/2019/6/27/mathematics-is-a-way-of-thinkinghow-can-we-best-teach-it

[6] Kris D. Gutiérrez. (2002). Studying Cultural Practices in Urban Learning Communities. Human Development 45, 312-321.

[7] Damir Jungic and Veselin Jungic. (2020). Dynamic Visual Models: Ancient Ideas and New Technologies, In From analysis to visualization: A celebration of the life and legacy of Jonathan M. Borwein, Callaghan, Australia, September 2017. Springer, 189-201.

[8] Nathan W. Klingbeil, Richard E. Mercer, Kuldip S. Rattan, Michael L. Raymer, and David B. Reynolds. (2005). "The WSU Model for Engineering Mathematics Education," Proceedings 2005 ASEE Annual Conference \& Exposition, Portland, Oregon, June 2005.

[9] Nathan W. Klingbeil, N. and Anthony Bourne. (2015). The Wright State Model for Engineering Mathematics Education: Longitudinal Impact on Initially Underprepared Students. Proceedings 2015 ASEE Annual Conference and Exposition, Seattle, WA (June 2015), 26.1580.1-11. 
[10] MAA = Mathematical Association of America. (2004). The Curriculum Foundations Project: Voices of the Partner Disciplines. https://www.maa.org/sites/default/files/pdf/CUPM/crafty/curriculum-foundations.pdf

[11] Ralph Modjeski, John A. L. Waddell, Gardner S. Williams, and Arthur N. Talbot. (1908). The teaching of mathematics to students of engineering. Science 28(710), 161-170.

[12] Elena Nardi (2016). Teaching Mathematics To Non-Mathematicians: What Can We Learn From Research On Teaching Mathematicians? Proceedings of the 13th International Congress on Mathematical Education, Hamburg, 24-31 July 2016. Available at: https://archive.uea.ac.uk/ m011/ICME13TSG2Paper(Nardi)270716.pdf

[13] Vanessa Oguamanam, Taneisha Lee, Tom McKlin, Zane Cochran, Gregory Abowd, and Betsy DiSalvo. (2020). Cultural Clash: Exploring How Studio-Based Pedagogy Impacts Learning for Students in $\mathrm{HCl}$ Classrooms. In Proceedings of the 2020 ACM Designing Interactive Systems Conference (DIS '20). Association for Computing Machinery, New York, NY, USA, 1131-1142. DOI:https://doi.org/10.1145/3357236.3395544

[14] Karen Saxe and Linda Braddy (2016). A common vision for undergraduate mathematical sciences programs in 2025. The Mathematical Association of America.

[15] Paola Valero, Ole R. Christensen, and Ole Skovsmose. (2009). Introduction: The MultiLayered Transitions of Knowledge Production and University Education in Science. In University Science and Mathematics Education in Transition. New York, NY: Springer, 1-16.

[16] Jeannette M. Wing (2006). Computational Thinking. Communications of the ACM 49 (3), 33-35 (2006). 